\title{
Redução de resíduos sólidos industriais a partir da simbiose industrial em uma indústria têxtil brasileira
}

\section{Thales Volpe Rodrigues ${ }^{1, *}$, Lo-Ruana Karen Amorim Freire Sanjulião ${ }^{2}$, Luis Maurício Martins de Resende ${ }^{1}$, Rui Tadashi Yoshino ${ }^{1}$ e Joseane Pontes ${ }^{1}$}

\author{
${ }^{1}$ Universidade Tecnológica Federal do Paraná (UTFPR). Rua Dr. Washington Subtil \\ Chueire, 330. Ponta Grossa-PR, Brasil (CEP 84017-220). *E-mail: \\ thales.volpe@hotmail.com. \\ ${ }^{2}$ Universidade do Estado de Minas Gerais (UEMG). Av. Juca Stockler, 1130. \\ Passos-MG, Brasil (CEP 37900-106).
}

Resumo. 0 aumento da geração de resíduos sólidos e os impactos ambientais causados por eles vêm sendo um assunto de preocupação mundial, diante disto as empresas procuram métodos para reduzir o desperdício gerado. 0 presente trabalho faz uma análise das sobras de espumas produzidas por uma indústria têxtil do sudoeste mineiro, sob a ótica da Ecologia Industrial e traz uma proposta de tratamento de resíduos por meio de uma de suas ferramentas, a Simbiose Industrial e Produção mais Limpa. Estes conceitos auxiliam na interação entre corporações para minimizar a disposição de materiais residuais com finalidade de dar outra destinação aos subprodutos ou eliminar os resíduos gerados no processo, não simplesmente a destinação final, mas fazer com que alguns destes sejam transformados em matéria prima para outros processos produtivos, desenvolvendo na empresa uma visão de que seus resíduos são oportunidade de novas receitas agregando valor, ganhando assim vantagens competitivas no mercado. Assim o objetivo deste trabalho é reduzir os resíduos sólidos a partir da Simbiose Industrial. Para levantamento do portfólio bibliográfico utilizou-se a metodologia PRISMA que possibilitou a elucidação do tema. Os resultados obtidos com a aplicação da simbiose industrial geraram uma receita média de $\mathrm{R} \$ 40.000,00$ por ano, além da redução de aquisição de matérias primas, devido à aplicação do conceito de produção mais limpa que reduziu a utilização de refugo no processo. Este trabalho salienta que atitudes ecologicamente corretas, além de garantir a sobrevivência de gerações futuras, podem gerar receita e aprimorar a produção.

Palavras-chave: Sustentabilidade; Ecologia Industrial; Resíduos Sólidos; Simbiose Industrial.
Recebido

$17 / 02 / 2020$

Aceito

$26 / 08 / 2020$

Disponível on line

$27 / 08 / 2020$

Publicado

$31 / 08 / 2020$

Acesso aberto

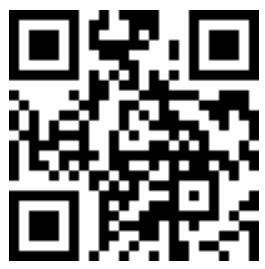

(D) 0000-0002-6102-3404

Thales Volpe

Rodrigues 


\begin{abstract}
Industrial solid waste reduction from industrial symbosis in a Brazilian textile industry. The increase in the generation of solid residues and the environmental impacts caused by them have been a matter of global concern, as companies are looking for methods to reduce the waste generated. The present work analyzes the leftover foams produced by a textile industry in the southwest of Minas Gerais State, Brazil, under the perspective of Industrial Ecology and presents a proposal for the treatment of waste through one of its tools, the Industrial Symbiosis and Cleaner Production. These concepts help in the interaction between corporations to minimize the disposal of residual materials in order to give another destination to the by-products or eliminate the residues generated in the process, not simply the final destination, but to make some of these be transformed into raw material for other productive processes, developing in the company a vision that its waste is an opportunity for new recipes adding value, thus gaining competitive advantages in the market. Thus, the objective of this work is to reduce solid residues based on Industrial Symbiosis. To survey the bibliographic portfolio, the PRISMA methodology was used, which made it possible to elucidate the theme. The results obtained with the application of industrial symbiosis generated an average revenue of $R \$ 40,000.00$ per year, in addition to the reduction in the acquisition of raw materials, due to the application of the cleaner production concept, which reduced the use of refuse in the process. This work stresses that ecologically correct attitudes, in addition to guaranteeing the survival of future generations, can generate revenue and improve production.
\end{abstract}

Keywords: Sustainability; Industrial ecology; Solid waste; Industrial symbiosis.

\author{
D) 0000-0002-3833-9646 \\ Lo-Ruana Karen \\ Amorim Freire \\ Sanjulião \\ (1) 0000-0003-4151-0843 \\ Luis Maurício Martins \\ de Resende \\ (D) 0000-0002-7267-4464 \\ Rui Tadashi Yoshino \\ D 0000-0002-0983-9705 \\ Joseane Pontes
}

\section{Introdução}

Nas últimas décadas os consumidores, cada vez mais, têm se preocupado com o desempenho ambiental dos produtos, obrigando as empresas a produzirem produtos ecologicamente corretos com baixo consumo de recursos (Du et al., 2017). No entanto, existem muitas deficiências nas soluções verdes tradicionais para os produtos, como, a abordagem de fim do tubo não pode eliminar poluentes ou resíduos, mas o que essa abordagem pode fazer é transformá-los de uma forma para outra, e as cadeias de suprimentos tradicionais reduzem o desperdício nos processos de fabricação e reutilizam produtos em fim de vida (Bansal e McKnight, 2009; Sheldon, 2018).

Devido ao crescente fluxo de resíduos sólidos gerados pelas organizações, o tema ambiental é debatido mundialmente. Assim a busca pela redução de seus impactos ambientais tem sido abordada constantemente, entretanto a gestão sustentável precisa estar vinculada ao conceito da sustentabilidade em todas suas áreas: econômica; social; ambiental (Despeisse et al., 2012; Ohnishi et al., 2012; Brunner e Rechberger, 2016; Queiroz e Vieira, 2018; Santos e Magrini, 2018). 
O crescimento populacional e seus padrões de vida, o aumento da urbanização das cidades e o rápido avanço tecnológico contribuíram para o aumento da quantidade e da variedade dos resíduos sólidos provenientes dos setores industrial, residencial e demais setores. Tendências mundiais apontam que o aumento do resíduo gerado pelos municípios entre 1980 a 1995 foi da ordem de $40 \%$, isto é, de $100 \mathrm{~kg}$ per capita para $140 \mathrm{~kg}$ per capita. Estima-se que até 2020 este número deve dobrar, apontando a necessidade de investimentos para que sejam criadas estações de tratamento, armazenagem e disposição de resíduos (OECD, 2017).

A atual conjuntura dos resíduos sólidos no Brasil ainda é precária, uma vez que, em $70 \%$ dos municípios brasileiros, 50,8\% dos resíduos sólidos ainda são dispostos em depósitos a céu aberto (lixões), com um volume de 30 milhões de toneladas por ano. Tal situação evidencia nada mais que a destinação de resíduos, reconhecidamente inadequada no Brasil (IBGE, 2017).

Os resíduos sólidos são gerados a partir de atividades de origem industrial e podem ser utilizados como matéria prima para outros fins. Diferentemente desta definição, lixo pode ser entendido como algo impossível de ser reaproveitado, e definido como inúteis. Assim, o termo resíduo sólido, está fundamentado na possibilidade de reutilização. Dessa forma, entende-se resíduo como matéria-prima em local (Andreoli et al., 2014; Paiva e Maria, 2018).

À interação meio ambiente, indústria, e sustentabilidade dá-se o nome de Ecologia Industrial (EI), que pode ser definida como uma estrutura emergente da gestão ambiental, a fim de transformar o sistema industrial, buscando encaixar as entradas e saídas com a capacidade local e planetária (Chertow e Park, 2016; Lowe e Evans, 1995). A EI busca integrar uma visão sistêmica entre o setor produtivo e o meio ambiente, com o objetivo de otimizar o uso dos recursos naturais. Concedendo ações em diferentes níveis de atuação para seu desenvolvimento (Chertow, 2000), seja ela dentro da empresa, entre empresas ou em escala regional.

A EI, vem sendo notada como uma ferramenta para reduzir os impactos ambientais causados pela produção industrial, sendo os conceitos de Produção mais Limpa (P+L) e Simbiose Industrial (SI) suas principais vertentes (Eckelman e Chertow, 2009). A P+L é a integração das estratégias técnica, econômica e ambiental aos processos e produtos, com 0 intuito de melhorar a eficiência no uso de matérias-primas, por meio da não geração, minimização ou reciclagem dos resíduos e emissões com benefícios ambientais, de saúde ocupacional e econômica (Fernandes et al., 2015). A partir de então, as organizações passam a ser responsabilizadas pela poluição ambiental, e seus processos passam por mudanças para reduzir o uso de recursos (Argenta, 2007).

Fernandes et al. (2001) definem a P+L como o emprego contínuo de uma estratégia econômica, para ampliar a eficiência na utilização de recursos, e aplicar a não geração dos mesmos. A partir deste conceito descrito pelo autor, a P+L supõe quatro atitudes básicas: Busca pela não geração de resíduos, por meio da racionalização das técnicas de produção; Minimização da geração dos resíduos; Reaproveitamento dos resíduos no próprio processo de produção; Reciclagem, com o aproveitamento das sobras ou do próprio produto para a geração de novos materiais. Dentro desta metodologia Maroun (2003), pergunta-se "onde estão sendo gerados os resíduos?" E "o que fazer com os resíduos gerados?". Dessa forma, evita-se o desperdício, tornando o processo mais eficiente.

Já a SI ocorre por meio de trocas físicas de materiais e produtos residuais entre indústrias, abordando a integração para a vantagem competitiva, melhorando assim o desempenho ambiental das empresas, bem como diminuindo seus custos totais (Chertow, 2000, 2007). Jacobsen (2006) define SI como a otimização de recursos e realização de intercâmbio de subprodutos e compartilhamento de benefícios entre as diferentes indústrias. 
Grant et al. (2010), relatam, que a interação mutualista entre diferentes indústrias no aproveitamento benéfico de resíduos ou energia resultam em uma produção mais eficiente em termos de recursos e menores impactos ambientais adversos. Chertow (2000) ressalta ainda, que para que aconteça a SI deve haver colaboração e oportunidades oferecidas por sinérgicos nas proximidades geográficas. Embora a localização não seja uma condição necessária, a proximidade geográfica é uma característica da SI.

Doménech e Davies (2011) explicam que a SI tem crescido com uma forte estrutura de intercâmbio para avançar para um sistema industrial mais eco eficiente, por meio do estabelecimento de uma rede colaborativa de intercâmbio de conhecimento, materiais e energia entre diferentes unidades organizacionais. A SI, portanto, tem como objetivo relacionar as empresas em um sistema coerente e inovador de vínculos e alianças organizacionais para reduzir o impacto ambiental da atividade industrial de uma forma economicamente racional (Doménech e Davies, 2011).

Mirata e Pearce (2006) salientam que as alianças da SI possibilitam melhorias na eficiência e eficácia dos diferentes recursos que são utilizados, demonstrando que os ganhos nas redes ou alianças têm sido mais vantajosos quando comparados às organizações que buscam melhorias fragmentadas em unidades individuais. Existem várias definições para o conceito, em resumo, a SI é baseada no compartilhamento de recursos e subprodutos entre as empresas (Bocken et al., 2012).

0 pioneiro e mais conhecido no conceito de SI, é o parque de Kallundborg, na Dinamarca. Somando 2,9 milhões de toneladas de materiais trocados, reduzindo coletivamente o consumo de água em $25 \%$ e abastecendo 5.000 casas com aquecimento urbano (Chertow, 2000). Devido a seu sucesso, é replicado em todo o mundo e esse modo de fabricação trouxe benefícios econômicos e ambientais significativos (Cao et al., 2018).

No Brasil, a Federação das Indústrias do Estado de Minas Gerais (FIEMG) coordena um programa de Simbiose Industrial denominado Programa Mineiro de Simbiose Industrial (PMSI). Esta iniciativa surgiu a partir do NISP e, apresenta-se como o único programa de Simbiose Industrial do Brasil realizado de forma sistêmica e sequencial. As práticas de SI brasileiras concentram-se principalmente em projetos que visam melhorar as práticas de sustentabilidade em clusters industriais (FIEMG, 2014).

De acordo com o Programa Nacional de Resíduos Sólidos (PNRS), até 2020, todos os resíduos sólidos industriais produzidos deverão ser corretamente destinados, incentivando a reciclagem e a cooperação entre os diferentes atores sociais, germinando a integração dos catadores de materiais recicláveis e valorizando as ações que envolvam a responsabilidade durante o ciclo de vida dos produtos (Schott e Vasconcelos, 2016).

Mesmo assim a atual conjuntura dos resíduos sólidos no Brasil ainda é precária, uma vez que, em $70 \%$ dos municípios brasileiros, 50,8\% dos resíduos sólidos ainda são dispostos em depósitos a céu aberto (lixões), com um volume de 30 milhões de toneladas por ano. Tal situação evidencia nada mais que a destinação de resíduos, reconhecidamente inadequada no Brasil (IBGE, 2017).

A empresa alvo do estudo gera em média setenta quilos de resíduos sólidos diariamente, que são coletados por uma empresa terceirizada, responsável pelo descarte apropriado. Assim o objetivo deste trabalho é reduzir os resíduos sólidos a partir da Simbiose Industrial. Para que o objetivo do estudo seja atingido, inicialmente realizou-se uma pesquisa, utilizando as bases de dados Scopus, Web of Science e Science Direct. Onde foi possível criar um portfólio relacionados ao tema Ecologia Industrial e suas ferramentas, permitindo assim a elucidação do tema e levantamento de ações importantes para a aplicação da SI. Para a filtragem dos artigos foi utilizada a metodologia de fluxograma PRISMA e snowball. 


\section{Metodologia}

Santos (2005) apresenta dois dos critérios básicos da classificação de pesquisa: quanto aos procedimentos técnicos utilizados pelo pesquisador e quanto aos objetivos pretendidos. Quanto aos objetivos, segundo a sistemática do autor, a pesquisa caracteriza-se pelo estudo descritivo, por se preocupar em relatar as atuais relações de troca de resíduos, frente às exigências do conceito de Simbiose Industrial de acordo com a literatura pesquisada.

0 estudo de caso é um estudo de natureza prática que analisa determinado fato, geralmente recente, dentro da realidade de um contexto, quando os limites entre o fato e $o$ contexto não são claramente definidos. Refere-se à análise aprofundada de um ou mais casos, para permitir o aprofundamento do conhecimento (Gil, 1996). Objetivando estimular a compreensão, sugerir hipóteses e questões ou desenvolver a teoria (Mattar, 1999). A principal inclinação dos estudos de caso, é tentar esclarecer por qual motivo uma decisão ou um conjunto de decisões foram tomadas, como foram aplicadas e com quais resultados alcançados (Yin, 2015).

O presente estudo caracteriza-se como uma pesquisa de campo ou de levantamento bibliográfico (Santos, 2005; Vergara e Peci, 2003). É bibliográfica devido à necessidade de estudo direto nas fontes científicas sobre o tema escolhido, como periódicos, artigos, livros, relatórios, dissertações e teses, dentre outras fontes, para desenvolver um mapeamento da literatura sobre o assunto. 0 estudo apresenta caráter quantitativo quanto aos resultados exibidos por tabulações, enquanto a solução do problema de pesquisa é estritamente qualitativa.

\section{Composição do portifólio bibliográfico}

A revisão de literatura se inicia com as definições dos eixos de pesquisa, que a princípio foram definidos como: Industrial Symbiosis (eixo 1) e solid waste (eixo 2). Em seguida foram definidas as palavras-chaves para pesquisa e suas variações. As combinações das palavras chave foram feitas por meio de operadores booleanos, que auxiliam na pesquisa possibilitando o uso de uma variedade maior de palavras.

As buscas dos artigos foram realizadas nas bases de dados: Scopus; Science Direct e Web of Science, por serem consideradas as mais relevantes para o tema. Para a construção do portfólio bibliográfico foram definidos filtros para delimitar de forma mais precisa a pesquisa. Optou-se então por realizar uma busca apenas por Article or Review, em que as palavras chave se encontrassem em Article title, Abstract, Keywords. Optou-se em não realizar o corte temporal para identificar os autores clássicos e artigos mais citados.

Para auxiliar durante todo o processo de filtragem foram utilizados os softwares de gerenciamento de referências, no qual possibilitou a formação do primeiro portfólio de artigos. A Tabela 1 apresenta o resultado das buscas nas bases de dados, assim como as combinações de palavras chave utilizadas.

Com a realização das buscas nas bases de dados, encontrou-se a quantidade de 574 , sendo a Scopus a base que retornou com uma maior quantidade de artigos, 311 documentos, que representa 54\% do portfólio total, em seguida a Web of Science com 174 artigos, 30\%, e por fim, a Science Direct com 89 artigos totalizando 16\%.

Tabela 1. Resultado das buscas nas bases de dados.

\begin{tabular}{|l|c|c|c|c|}
\hline Keyword combination & SC & WoS & SD & Total \\
\hline "Industrial Symbiosis" AND "solid waste" & 29 & 16 & 12 & 54 \\
\hline "Industrial Symbiosis" AND (residue OR waste OR leavings) & 282 & 158 & 77 & 517 \\
\hline & $\mathbf{3 1 1}$ & $\mathbf{1 7 4}$ & $\mathbf{8 9}$ & $\mathbf{5 7 4}$ \\
\hline
\end{tabular}

Rev. Bras. Gest. Amb. Sustent., 2020, vol. 7, n. 16, p. 835-853. 
Após a etapa de busca, iniciou-se a etapa de filtragem dos artigos, conforme a metodologia PRISMA. Os artigos identificados na fase de busca foram excluídos conforme: Duplicatas, Leitura de títulos, leitura de resumos e leituras na integra. Para a excluir as duplicidades de artigos foram utilizados os softwares Mendeley Desktop® e JabRef®. Durante a leitura dos trabalhos foram identificadas referências relevantes para a pesquisa, que não foram apresentadas na fase de busca nas bases de dados, estes foram inclusos ao portfólio, conforme orienta a metodologia snowball (Goodman, 1961), obtendo assim, um portfólio final de 31 artigos, que foram lidos de forma integral para o esclarecimento do tema para iniciar o estudo de caso. A Figura 1 apresenta as fases de filtragem dos artigos, utilizando o fluxograma PRISMA.

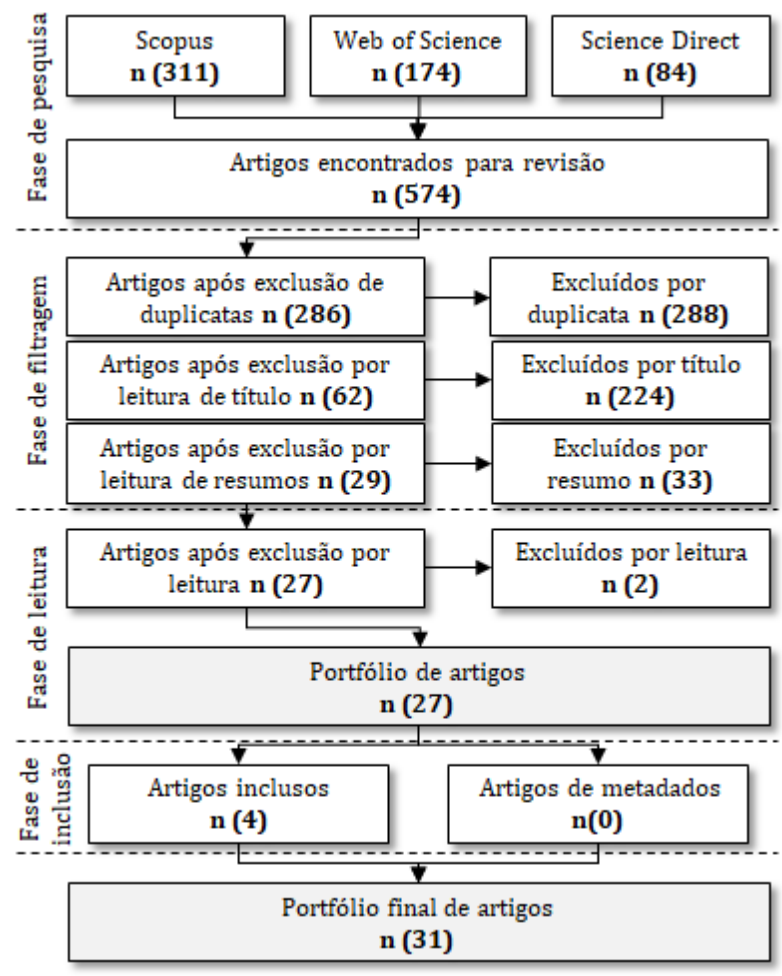

Figura 1. Fase de exclusão para composição de portfólio de artigos. Fonte: Adaptado de Moher et al. (2009).

A leitura dos artigos do portfólio final proporcionou aos autores o conhecimento necessário para atingir o objetivo da pesquisa. Onde foi levantado os aspectos a respeito dos trabalhos referentes à Ecologia industrial, Produção mais limpa e principalmente Simbiose Industrial. Por meio desta pesquisa, pôde-se perceber o grau de importância da aplicação deste conceito na eliminação de resíduos. Observou-se, ainda, a carência de pesquisas sobre a implantação de tal conceito em caráter, o que motivou a realização do presente trabalho.

\section{Fases do estudo de caso}

Para que o objetivo fosse alcançado, este trabalho foi estruturado quanto as etapas descritas por Miguel (2007). Dividido em seis etapas, a proposta para se conduzir uma 
pesquisa por meio de um estudo de caso é relacionada às atividades apresentadas na Tabela 2.

Tabela 2. Proposta para se conduzir uma pesquisa por meio de um estudo de caso.

\begin{tabular}{|l|l|}
\hline Atividade & Descrição \\
\hline $\begin{array}{l}\text { Definir uma estrutura } \\
\text { conceitual teórica }\end{array}$ & $\begin{array}{l}\text { Mapear a literatura acerca do tema, delinear as proposições, delimitar as } \\
\text { fronteiras e grau de evolução; }\end{array}$ \\
\hline Planejar os casos & $\begin{array}{l}\text { Selecionar as unidades de análise e contatos, escolher os meios para } \\
\text { coleta e análise dos dados, definir meios de controle da pesquisa; }\end{array}$ \\
\hline Conduzir teste piloto & $\begin{array}{l}\text { Testar procedimentos de aplicação, verificar a qualidade dos dados, fazer } \\
\text { os ajustes necessários; }\end{array}$ \\
\hline Coletar os dados & Contatar os casos, registrar os casos, limitar os efeitos do pesquisador; \\
\hline Analisar os dados & $\begin{array}{l}\text { Produzir as narrativas, reduzir os dados, construir painel, identificar } \\
\text { causalidade; }\end{array}$ \\
\hline Gerar relatório & Desenhar implicações teóricas, prover estrutura para replicação. \\
\hline
\end{tabular}

Fonte: Adaptado de Miguel (2007)

(1) Definição de uma estrutura conceitual teórica: Esta fase implica em pesquisas e embasamentos nas diversas literaturas referentes ao tema a ser abordado nos trabalhos científicos. Os principais meios de pesquisas foram: artigos, monografias, sites, normas, leis, entre outros.

Então, foi feito um levantamento bibliográfico a respeito dos trabalhos referentes à Ecologia industrial, Produção mais limpa e principalmente Simbiose Industrial. Por meio desta pesquisa, pôde-se perceber o grau de importância da aplicação deste conceito na eliminação de resíduos. Observou-se, ainda, a carência de pesquisas sobre a implantação de tal conceito em caráter, o que motivou a realização do presente trabalho.

(2) Planejamento do caso: Nesta fase definiu-se o caso a ser estudado e o levantamento dos problemas em torno do método atual de simbiose industrial, demonstrando de maneira clara a razão para se desenvolver o modelo do estudo. Nesta fase definiram-se também outros objetivos desejados pelo pesquisador para que o modelo proposto pudesse alcança-los ao final do seu processo.

(3) Conduzir teste piloto: Esta fase não foi realizada por se tratar de um estudo de caso único, dispensando a realização do mesmo.

(4) Coleta de dados: A coleta de dados foi realizada pelo autor por meio de observação visual no local do estudo, onde as informações coletadas foram adicionadas a uma planilha do Excel, que foram ligadas à históricos provenientes de planilhas de controles produtivos utilizados pelo setor de produção.

Para mapeamento do processo, de forma a identificar os setores geradores de resíduo, foi utilizada a ferramenta IDEF-0, com dados coletados na observação e entrevistas convencionais feita com colaboradores, sendo as principais perguntas sobre 0 processo de geração de resíduos e os próprios resíduos gerados.

Por meio de coleta de dados levantou-se as características da empresa estudada, para a identificação de possíveis parcerias para o resíduo de espuma, dando preferência a interações mais próximas. Com o intuito de fortalecer o conceito da Simbiose Industrial para os resíduos sólidos. A Figura 2 apresenta o fluxograma com passo a passo desta fase da pesquisa. 


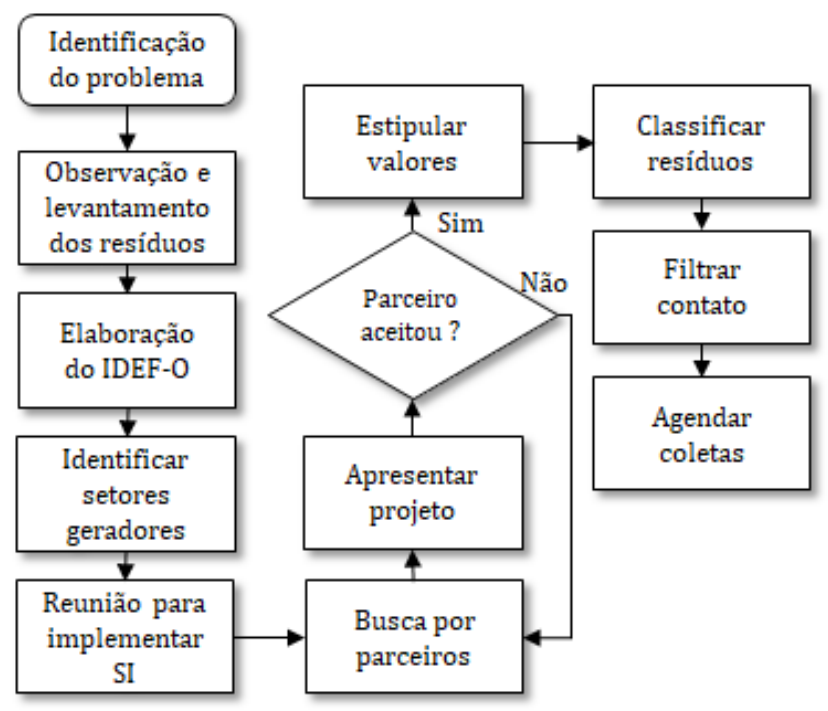

Figura 2. Fluxograma para planejamento do caso.

(5) Analisar os dados: Após a coleta dos dados, foi feita uma filtragem dos dados com o objetivo de se obter apenas os que fossem relevantes para a condução do estudo, como os resíduos reaproveitáveis e a classificação dos mesmos. Com essa filtragem o número de resíduos gerados, identificação de tipos de resíduos, custo de descarte e desenvolvimento de ações para aplicação da $\mathrm{P}+\mathrm{L}$, com intuito de reduzir a geração de resíduo na fonte, foram apresentadas.

Para o levantamento do problema, este trabalho focou em reuniões com os envolvidos para listar os objetivos, potenciais parcerias, dificuldades e atitudes a concretizar para a implantação da Simbiose Industrial. Utilizando a metodologia de ecologia industrial e produção mais limpa foram definidas as atitudes a serem tomadas para reduzir os resíduos na fonte, com alterações na ficha técnica e adequação da matéria prima. A Figura 3 apresenta o fluxograma com o passo a passo desta fase da pesquisa.

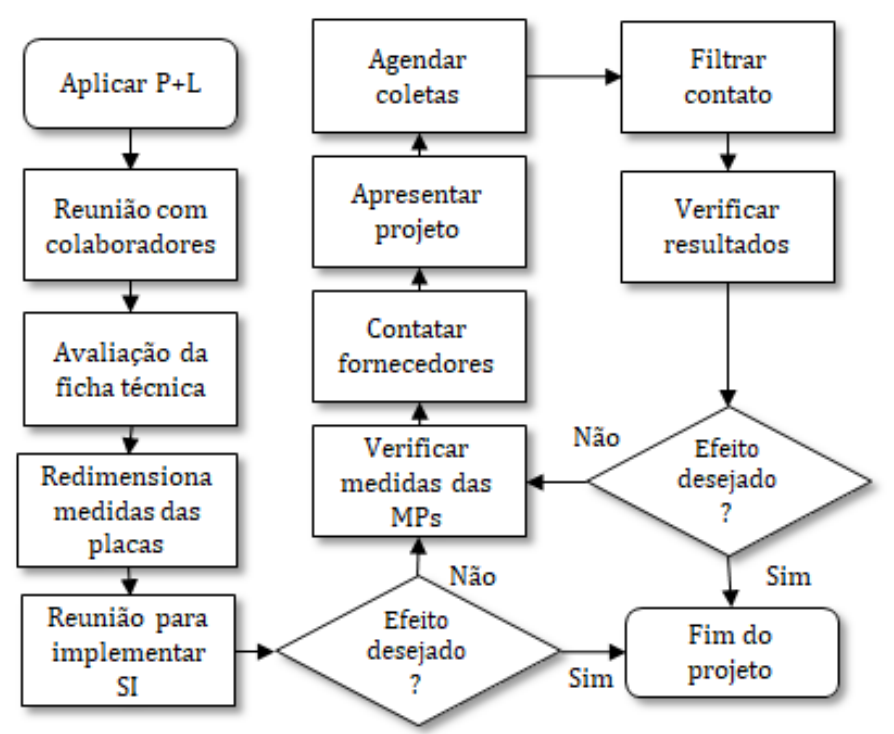

Figura 3. Fluxograma para análise de caso. 
(6) Gerar relatório: Por fim, por meio de planilhas dos softwares Excel e MS Project, e de forma textual do presente trabalho, foram apresentados os resultados obtidos na aplicação da SI e P+L na organização. Onde se apresentou os benefícios tanto ambientais quanto monetários.

O Microsoft Project é um software desenvolvido pela Microsoft para o gerenciamento de projetos. Esse aplicativo possibilita organizar a informação sobre a atribuição de tempos às tarefas, a associação de custos tanto de mão de obra quanto de materiais, de forma a propiciar o gerenciamento dos prazos, sem exceder o orçamento, objetivando alcançar as metas propostas para o projeto (Rodrigues et al., 2019).

\section{Resultados e discussão}

O estudo proposto foi desenvolvido em uma indústria têxtil, no sudoeste de Minas Gerais e foi escolhida devido a abertura para coleta de dados, assim, favorecendo o desenvolvimento da pesquisa, além disso, o setor vem se destacando no cenário econômico nacional, trazendo oportunidades para melhorias em diversas áreas.

A fábrica se caracteriza como Média Empresa e sua administração é familiar, onde o proprietário é também o diretor geral e tem grande participação nos processos administrativos, comerciais e de produção. Possui separadamente o setor comercial, compras, administrativo, planejamento e produção.

Atualmente se encontra em expansão, com demanda para exterior, possui um espaço físico de aproximadamente $5.000 \mathrm{~m}^{2}$ e conta com 120 funcionários com produção média de 800.000 de pares de bojo mensais. 0 processo produtivo é dividido em nove setores, sendo eles almoxarifado, dublagem, corte, prensa, balancim, revisão, montagem, expedição e resíduos.

Identificação do problema. A empresa não possui dados históricos relacionados à quantidade de resíduo gerado, então o índice de resíduos foi calculado por informações disponibilizadas pelos gestores para elaborar uma planilha, no Excel, para calcular a média de resíduos gerados no processo.

De acordo com dados, o rendimento médio da empresa é de 25 pares por metro de matéria prima, a produção mensal é de 800.000 pares, portanto a mesma utiliza cerca de $32.000 \mathrm{~m}$ de espuma por mês, que corresponde a $6.400 \mathrm{~kg}$. Em uma média de 20 dias uteis a empresa utiliza 320 quilogramas por dia, porém $22 \%$ desta matéria prima era considerada resíduo, segundo cálculos realizados com modelos de maior demanda, gerando cerca de $70 \mathrm{~kg}$ de resíduo de espuma diariamente. Esse resíduo era coletado por terceiros e descartadas em local apropriado. 0 serviço prestado custava em média $\mathrm{R} \$ 400,00$, o que totalizava em média $\mathrm{R} \$ 1.600,00$ mensais.

Observação e levantamento dos resíduos do processo. 0 índice médio de resíduos gerados foi calculado a partir de dados dispostos pelo responsável de criação dos moldes. Cada molde possui uma ficha técnica, onde constam as medidas do mesmo, a partir de então foi avaliado com base no histórico de produção e encontrada a média de resíduo gerado. De acordo com históricos da empresa, foi realizado um gráfico de Pareto (Figura 4) onde se encontrou que alguns modelos representaram mais de $80 \%$ da produção de 2017, portanto esses modelos foram utilizados para encontrar uma média de resíduo gerado. 


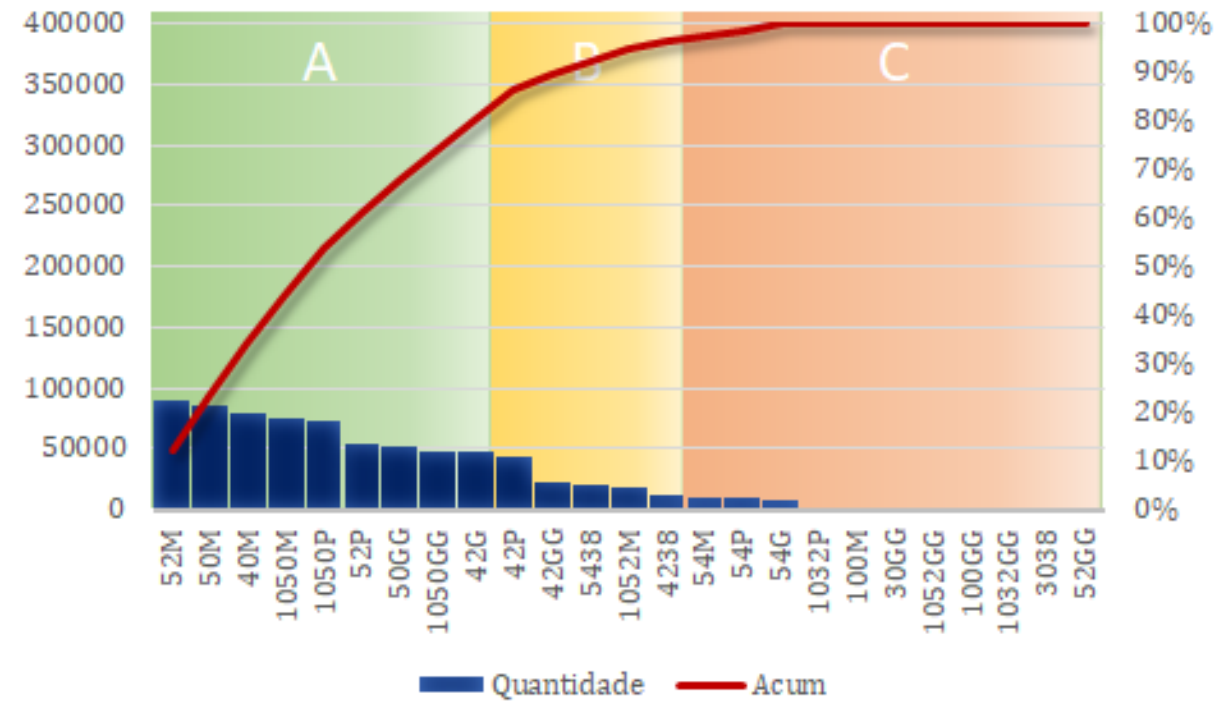

Figura 4. Gráfico de Pareto.

Por meio de observações no processo e entrevistas com funcionários também foi possível mapear a empresa. Nesta fase, os setores geradores de sobras de espumas foram identificados. Em seguida foi realizada uma avaliação em todo o processo produtivo e levantados os tipos de resíduos sólidos resultantes das atividades produtivas.

0 processo de mapeamento iniciou-se no setor 1 , onde o tecido é colado na espuma em um processo de queima, o qual não possui resíduos sólidos significativos, em seguida no setor 2, onde essa espuma em união do tecido, denominado manta, é cortado nas medidas de cada modelo, chamado placa, este processo emite um resíduo denominado "refugo de espuma" (Figura 5), que é gerado devido a medida não ser suficiente para transformar em uma placa inteira.

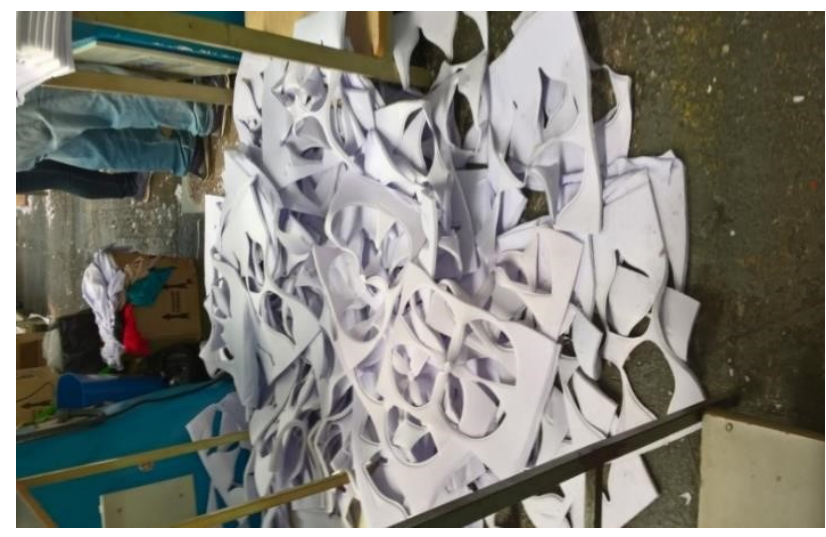

Figura 5. Refugo de espuma.

O setor 3 molda estas placas e também não gera resíduos, por fim o setor 4 realiza o corte dos moldes para transformação do produto final, bojo. Este último processo 
origina-se o resíduo de maior representatividade da empresa, as "rebarbas da placa moldada" da qual o bojo é destacado, apresentados pela Figura 6.

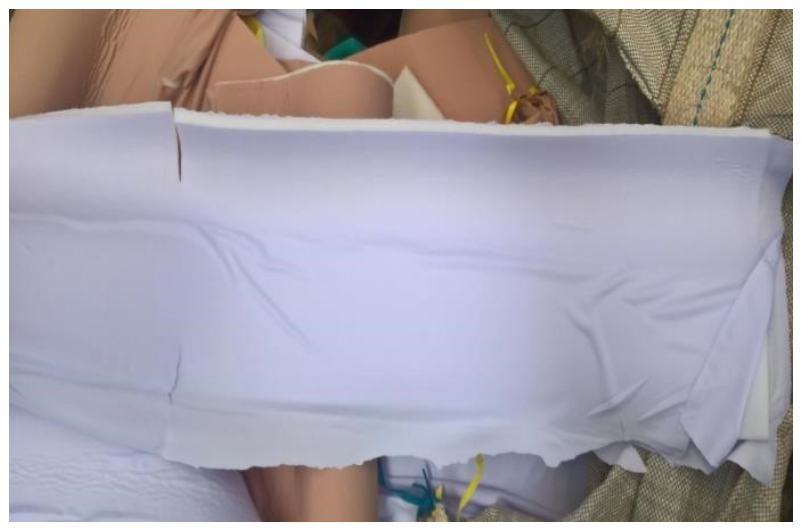

Figura 6. Rebarba de espuma.

A partir destes dados foi possível elaborar o IDEF-0 do processo. Representado pela Figura 7. Como pode-se observar no IDEF-0, o setor de corte gera o resíduo denominado refugo de espuma, que se dá a partir do processo de corte de placas, onde são cortadas as placas na medida pré-estabelecida de acordo com a ficha técnica, uma sobra na lateral da espuma não é aproveitada devida ao tamanho inferior ao desejado. Outro setor que apresenta geração de resíduo é o setor de balancim, que gera a rebarba de espuma, onde é destacado o bojo da placa moldada.

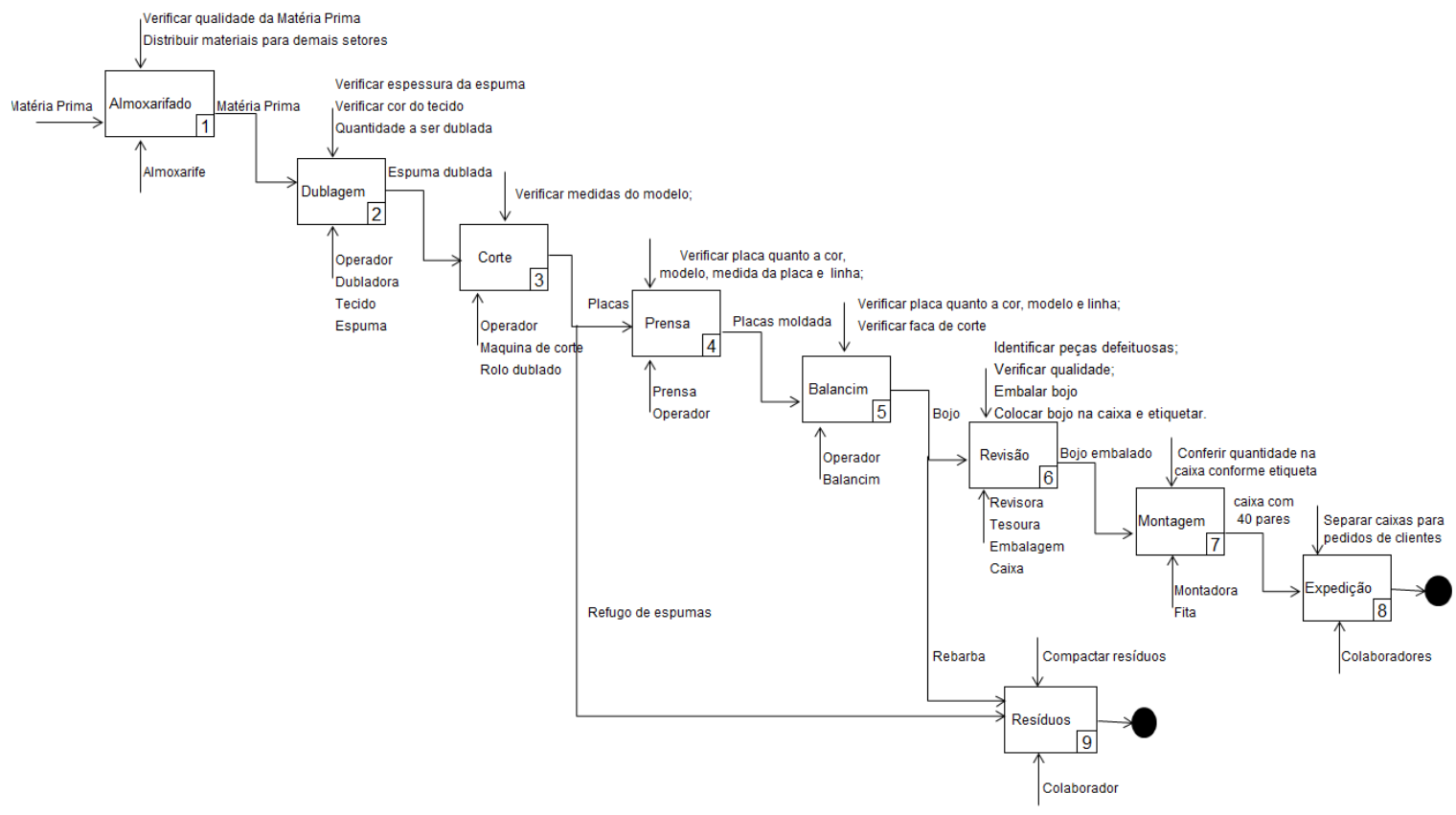

Figura 7. IDEF-0 do processo.

Rev. Bras. Gest. Amb. Sustent., 2020, vol. 7, n. 16, p. 835-853. 
Reunião para implantação da SI. A Ecologia industrial vem sendo um dos sistemas mais comentados atualmente com a ideia do fluxo ideal de resíduos em um sistema industrial, onde todos os resíduos são aproveitados ao máximo, levando à empresa a emissão zero. No contexto da SI às expectativas conceituais, marcadas pela ajuda mútua entre organizações, que intercambiam resíduos como resultados positivos, tanto na redução dos impactos ambientais quanto na geração de vantagem competitiva para os participantes.

Após identificados e levantados os dados necessários, foi realizada uma reunião com o diretor, coordenadores, líderes de setores e colaboradores para apresentar os seguintes tópicos:

(1) Conceito de SI. Para dar início ao projeto de aplicação da SI foi ministrado aos colaboradores e diretoria uma pequena apresentação de slides sobre a SI, com o intuito de apresentar o conceito e esclarecer demais dúvidas geradas durante a apresentação. Após todos estarem empolgados com o conceito de SI foi apresentada uma proposta de aplicação da SI, onde os pesquisadores e colaboradores buscariam parceiros para realizar o intercâmbio de sobras de espumas, a fim de eliminar o descarte das sobras de espumas e gerar receita para a empresa. Mediante a aprovação da diretoria foi realizado uma planilha para gerenciar o andamento do projeto, onde foram delegadas funções para cada colaborador e prazos para cada ação;

(2) Parceiros para SI. Com a equipe SI já formada, foram apresentados os possíveis parceiros. Foram avaliadas 6 empresas que utilizam espuma como matéria prima, entre elas, 1 empresa de pelúcia, 3 de estofados, 1 de colchões e 1 de travesseiros e almofadas, todas geograficamente próximas. Após esta avaliação os representantes da empresa entraram em contato com as possíveis parceiras para apresentar o projeto e questionar sobre o interesse de fazer parte do mesmo. Das 6 empresas identificadas 4 se interessaram em conhecer o projeto, sendo 2 de estofado, 1 de colchão e 1 de almofada. Por fim uma reunião foi marcada com todas as parceiras para apresentar o conceito SI;

(3) Classificação dos resíduos. Após identificados os setores geradores de resíduos e encontrados os parceiros para intercâmbio da SI, as sobras de espumas foram classificadas, e os códigos para informação interna foram criados e apresentados às parcerias para identificar a utilidade para as mesmas.

Os fabricantes de estofados e almofadas utilizam qualquer espuma, pois a mesma é triturada e colocada como recheio do novo estofamento e almofadas. Já o fabricante de colchão adiciona o resíduo ao processo de formação da espuma e tem a necessidade de espumas mais claras por questão de estética, portanto as sobras de espumas mais claras foram priorizadas para o mesmo.

(4) Definição dos valores. Por fim houve a negociação de valores do resíduo, entre o diretor, gerente, parceiros e o autor, isto para evitar um valor desproporcional que poderia inviabilizar o projeto. Após definido o preço viável e lucrativo para todos (Tabela 3), o contrato foi firmado. Ainda pode-se destacar a eliminação de resíduos na empresa estudada e redução na compra de matéria prima para os parceiros. 
Tabela 3. Valor dos resíduos sólidos.

\begin{tabular}{|c|c|c|c|}
\hline Cód. do resíduo & Tipo de Resíduo & Condição & Valor R\$ (kg) \\
\hline LCC01 & Limpo cores claras & Limpo & $\mathrm{R} \$ 1,50$ \\
\hline LCE01 & Limpo cores escuras & Limpo & $\mathrm{R} \$ 1,50$ \\
\hline ILC02 & Impurezas Leves claras & Leve impureza & $\mathrm{R} \$ 1,20$ \\
\hline ILE02 & Impurezas Leves escuras & Leve impureza & $\mathrm{R} \$ 1,20$ \\
\hline SCC03 & Sujo cores claras & Sujo & $\mathrm{R} \$ 1,00$ \\
\hline SCE03 & Sujo cores Escuras & Sujo & $\mathrm{R} \$ 1,00$ \\
\hline
\end{tabular}

Ainda pode-se destacar a eliminação de resíduos na empresa estudada e redução na compra de matéria prima para os parceiros. As matérias primas adquiridas pelos parceiros custam em média $\mathrm{R} \$ 1,40$ por metro, 1 metro pesa cerca de 200 gramas, ou seja, $\mathrm{R} \$ 6,98$ por quilo, a indústria de estofado e almofada utiliza todos os tipos de resíduos que equivale em média $\mathrm{R} \$ 1,23$ o quilo, ou seja uma economia de $82 \%$ no preço da matéria prima, porém cada parceiro utiliza apenas uma parcela do resíduo no processo. Assim, cada parceiro informou sua previsão de demanda mensal, em quilogramas, e qual a porcentagem utilizada no processo, coluna 3 da Tabela 4.

Tabela 4. Demanda das empresas.

\begin{tabular}{|l|c|c|c|c|c|}
\hline \multicolumn{1}{|c|}{ Parceiro } & $\begin{array}{c}\text { Demanda } \\
\text { mensal (kg) }\end{array}$ & Utilização & $\begin{array}{c}\text { Demanda de } \\
\text { resíduo (kg) }\end{array}$ & Valor por kg & Total \\
\hline Estofado 1 & 950 & $50 \%$ & 475 & $\mathrm{R} \$ 1,23$ & $\mathrm{R} \$ 584,25$ \\
\hline Estofado 2 & 800 & $50 \%$ & 400 & $\mathrm{R} \$ 1,23$ & $\mathrm{R} \$ 492,00$ \\
\hline Colchões & 650 & $30 \%$ & 195 & $\mathrm{R} \$ 1,50$ & $\mathrm{R} \$ 292,50$ \\
\hline Almofadas & 480 & $100 \%$ & 480 & $\mathrm{R} \$ 1,23$ & $\mathrm{R} \$ 590,40$ \\
\hline & $\mathbf{2 . 8 8 0}$ & $\mathbf{5 8 \%}$ & $\mathbf{1 . 5 5 0}$ & $\mathbf{R} \$ \mathbf{1 , 3 0}$ & $\mathbf{R} \mathbf{1 . 9 5 9 , 1 5}$ \\
\hline
\end{tabular}

De acordo com o contrato as parcerias são responsáveis por coletar os resíduos da espuma, e a empresa estudada fornecer a mão de obra para carregar os caminhões. De acordo com essa demanda encontrada as coletas foram agendadas, onde cada parceiro escolheu o melhor dia da semana para realizar a coleta (Tabela 5).

Tabela 5. Agendamento de coletas.

\begin{tabular}{|l|c|c|c|c|c|}
\hline & $\begin{array}{c}\text { Resíduo de espuma } \\
\text { (média) }\end{array}$ & Terça feira & Quarta feira & Quinta Feira & Sexta feira \\
\cline { 3 - 6 } & 210 & Estofado 1 & Estofado 2 & Colchões & Almofadas \\
\hline Semana 1 & 350 & 0 & 0 & 0 & 210 \\
\hline Semana 2 & 350 & 125 & 100 & 0 & 125 \\
\hline Semana 3 & 350 & 125 & 100 & 100 & 0 \\
\hline Semana 4 & 350 & 125 & 100 & 100 & 0 \\
\hline Semana 5 & $\mathbf{1 . 6 1 0}$ & $\mathbf{4 7 5}$ & $\mathbf{4 0 0}$ & $\mathbf{2 0 0}$ & $\mathbf{4 8 5}$ \\
\hline Total & \multicolumn{5}{|c|}{$\mathbf{1 . 5 6 0}$} \\
\hline
\end{tabular}


(5) Atitudes mais limpas. A quantidade demandada mensalmente pelas parceiras era inferior a quantidade gerada pela empresa. A partir desta análise foi elaborado um novo projeto para reduzir o resíduo gerado na fonte, utilizando o conceito da $\mathrm{P}+\mathrm{L}$. Para que isso fosse possível foi realizada mais uma reunião onde foi apresentado aos colaboradores o conceito da $\mathrm{P}+\mathrm{L}$.

Durante a reunião ficou aberto para todos os presentes expressarem suas opiniões, onde o contexto principal foram os conceitos de EI. A ficha técnica foi reavaliada e notouse que algumas placas poderiam ser redimensionadas e reduzir o seu tamanho. Esse processo de redução das placas foi avaliado em conjunto com o responsável pelo desenvolvimento para averiguar se as mudanças não afetariam a qualidade do produto, após a sua aprovação a equipe designada ficou responsável em alterar a ficha técnica e comunicar aos líderes de produção sobre a mudança, por meio de e-mails e comunicados informativos.

A nova ficha técnica reduziu a geração de resíduo de espuma de $22 \%$ para $18 \%$ ao mês, porém não foi suficiente. Atingindo assim a fase 2 de aplicação da SI, na qual as empresas se preocupam com a geração de resíduos nos processos produtivos, e elaboram ações para elimina-la na fonte por meio de novas tecnologias ou de gestão empresarial.

A partir de então foi observado que o resíduo "refugo de espuma" só era gerado devido ao tamanho da espuma utilizada ser inferior a medida necessária para fazer 4 placas, a Tabela 6 apresenta a comparação da medida real com a ideal, (os cálculos foram realizados com base nos produtos que representam $80 \%$ da demanda).

Tabela 6. Medida ideal da matéria-prima.

\begin{tabular}{|c|c|c|c|}
\hline & Largura da placa & Largura da espuma & Placas \\
\hline Real & $0,42 \mathrm{~m}$ & $1,60 \mathrm{~m}$ & 3,8 \\
\hline Ideal & $0,42 \mathrm{~m}$ & $1,68 \mathrm{~m}$ & 4,0 \\
\hline
\end{tabular}

Para resolução deste problema os fornecedores de espuma foram contatados para verificar se existia a matéria com $1.68 \mathrm{~m}$, que informaram que a medida da forma de "espumação" é no máximo $1.70 \mathrm{~m}$, porém passa por um processo de "refilação" para retirar as "cascas" de espuma e impurezas do processo, onde é retirado $5 \mathrm{~cm}$ de cada lateral da espuma, deixando-a com $1.60 \mathrm{~m}$. Posteriormente a esta informação foi solicitado aos fornecedores amostras de espuma sem refilar para realização de testes no processo, no qual se percebeu que a espuma sem refilar atendia as necessidades de medida.

Foi solicitado aos fornecedores que abastecessem a empresa apenas com espumas sem refilar, o mesmo não demonstrou dificuldades em realizar a solicitação, visto que eliminaria um processo de fabricação e também reduziria a quantidade de resíduos gerada por eles. Atingindo a 3 a fase da aplicação da SI, onde as empresas percebem que muitas das soluções para a eliminação de resíduos são insuficientes e utilizam um resíduo para outro segmento produtivo.

0 refugo de espuma que atualmente é gerado apenas por modelos de menor demanda está sendo produzido em ferramentas menores, desenvolvidas com 1 ou 2 pares, e foi criado um setor denominado aproveitamento que utiliza placas fora da medida para confeccionar pares nas cores desejadas. Um mês após a criação do setor de aproveitamento, onde sobras de espumas, antes descartadas são transformadas em produtos para comercialização, foram produzidos 4.238 pares, que anteriormente eram tratados como resíduos. 


\section{Resultados da simbiose industrial}

A simbiose industrial é apresentada por muitos autores como um modelo a ser seguido, visto que os resultados alcançados, incluem ganhos econômicos, ambientais e sociais. Os resultados da cooperação e integração das empresas participantes, em busca de economia dos recursos naturais, e também da possibilidade de maiores ganhos econômicos, sem impedir o aumento da produção, podem levar as organizações participantes ao crescimento e a introdução de novas tecnologias quando necessário.

Como resultado, a empresa teve sua imagem melhorada, pois eliminou o descarte de sobras de espumas no meio ambiente, aumentou sua performance ambiental, e teve um aumento significativo da receita, estimado em cerca de $\mathrm{R} \$ 40.000$ por ano. No mês inicial da SI, foram intercambiados $1.550 \mathrm{~kg}$ de resíduos de espumas, por cerca de $\mathrm{R} \$ 1,30 \mathrm{o} \mathrm{kg}$ para os aliados do projeto, o setor de aproveitamento comercializou $\mathrm{R} \$ 1.597,24 \mathrm{em}$ produtos que anteriormente eram descartados, totalizando $\mathrm{R} \$ 3556,39$. Prevê que ao discorrer dos 12 meses a receita da empresa chegue a $R \$ 42.676,68$, porém estimasse que os ganhos com a SI aumentem de acordo com desenvolvimento do projeto.

A despesa $\mathrm{R} \$ 1.600,00$ resultante das coletas realizadas por uma empresa terceirizada foram suspensas. A matéria prima, atualmente com $1.70 \mathrm{~m}$, reduziu o nível de refugo de espuma e também geram receita, uma vez que o rendimento em pares por metro aumentou de 24 pares/metro para 32 pares/metro, nos modelos de maior demanda. A organização do barracão melhorou e os colaboradores aderiram bem ao conceito de SI, após o treinamento.

\section{Considerações finais}

O desenvolvimento econômico, baseado no modo de produção capitalista, resultado da revolução industrial, trouxe consequências danosas à sociedade e ao meio ambiente, como o problema do lixo. A utilização de áreas do conhecimento como a gestão de operações, as engenharias, a ecologia, entre outros, contribui para solucionar problemas que por origem já trazem certa complexidade. Assim, para transformar um modo de produção e consumo convencional, para uma realidade em que a sustentabilidade seja a principal meta, é necessário quebrar com algumas estruturas e pensamentos fragmentados.

Este trabalho apresentou o conceito de Ecologia Industrial, com o objetivo de aplicar um de seus instrumentos, a Simbiose Industrial, na redução de resíduos sólidos, redução de impactos ambientais e obtenção de lucros.

Para atingir o objetivo geral o estudo se iniciou com a observação no processo para elaboração do IDEF-0, onde os setores geradores de resíduos foram identificados, em seguida um plano de ação foi elaborado, no MS Project, para controlar as ações do projeto. Os colaboradores foram treinados e se iniciou a busca por parceiros para associar ao projeto, que apresentou vantagens para tanto para os possíveis parceiros quanto para a organização estudada.

Assim a aplicação da SI se caracteriza em um modelo auto organizado, no qual um ecossistema industrial emerge de decisões feitas por atores privados motivados pelas trocas de recursos para atingir metas, tais como: redução de custos, aumento da receita, e/ou expansão dos negócios, que se manifestou de forma independente do tipo bottom-up, a partir de sinergias auto iniciadas em redes de Simbiose Industrial coordenadas pelos próprios participantes.

Em seguida foi elaborado um novo plano para reduzir os refugos de espuma gerados no processo, onde a ficha técnica foi alterada para otimizar a utilização de matéria prima, foi quando se percebeu que a inadequação da matéria prima era a principal responsável pela geração do resíduo. Por fim os fornecedores foram contatados e após as 
reuniões e as apresentações do projeto o recurso foi adequado para o processo da empresa alvo de estudo, sendo lucrativo para ambos.

Em princípio os colaboradores da empresa e possíveis parceiros ficaram desconfiados com o conceito, porém após a apresentação do mesmo as incertezas foram erradicadas, alguns possíveis parceiros não aderiram ao projeto, pois ficaram com receio de afetar a qualidade de seus produtos com a utilização de resíduos. Outra dificuldade encontrada foi a de avaliar os valores de resíduos gerados, uma vez que a empresa não possuía histórico da geração do mesmo. A demanda solicitada pelo parceiro também pode ser uma barreira, porque tanto o resíduo gerado quanto o utilizado para SI é sazonal e pode faltar ou sobrar para intercambiar.

Apesar das barreiras encontradas os benefícios foram bem satisfatórios, gerando receita de aproximadamente $R \$ 3.300,00$ mensais para a empresa estudada, além de eliminação de despesas com coletas de resíduos, resultando na eliminação de resíduos depostos no ambiente. Com a aplicação da $\mathrm{P}+\mathrm{L}$ o rendimento da matéria prima teve um aumento de $22 \%$, além de reduzir os resíduos dos fornecedores.

Em tempos que a sustentabilidade e a prevenção ao meio ambiente vêm sendo centro de discussões mundiais, este trabalho demonstra que a utilização do conceito ecologia industrial e seus instrumentos, principalmente a SI, é uma importante ferramenta na eliminação de resíduos, aliada com a redução na fonte, evidenciando que atitudes sustentáveis também podem ser lucrativas, além de outros benefícios.

Recomenda-se para trabalhos futuros a aplicação da $4^{\underline{a}}$ fase, adesão de novos membros para a Simbiose Industrial, onde as empresas divulgam o conceito da SI e os resultados alcançados e se organizam para desenvolver parcerias com novos membros, que sozinhos não tem condições técnicas e econômicas para se manter nesse processo. Nessa fase há também um intercâmbio de conhecimentos e práticas adquiridas com outras empresas. E a fase 5, atração de novos empreendimentos para o parque industrial, que é quando as empresas atraem novos empreendimentos (produtos e serviços) para esse cenário, envolvendo a participação de órgãos governamentais e de incentivo à implantação de empreendimentos na região, pois a implantação da SI em um polo industrial traz, entre outros, o benefício do melhor uso de recursos naturais, gerando assim um avanço significativo nos âmbitos econômicos, ambientais e sociais.

\section{Conflito de interesses}

Os autores declaram não haver conflito de interesses.

\section{Referências}

Andreoli, C. V.; Andreoli, C. V.; Andreoli, F. N.; Trindade, T. V.; Hoppen, C. Resíduos sólidos: origem, classificação e soluções para destinação final adequada. In: FAEP - Federação da Agricultura do Estado do Paraná. Complexidade: redes e conexões do ser sustentável. 1. ed. Curitiba: FAEP, 2014. (Coleção Agrinho). p.31-52. Disponível em: <https://www.agrinho.com.br/materialdoprofessor/residuos-solidos-origemclassificacao-e-solucoes-para-destinacao-final-adequada>. Acesso em: 21 fev. 2020.

Argenta, D. O. F. Alternativas de melhorias nos processos produtivos do setor moveleiros de Santa Maria/RS: impactos ambientais. Santa Maria: Universidade Federal de Santa Maria, 2007. (Dissertação de mestrado).

Bansal, P.; Mcknight, B. Looking forward, pushing back and peering sideways: Analyzing the sustainability of industrial symbiosis. Journal of Supply Chain Management, v. 45, n. 4, p. 26-37, 2009. https://doi.org/10.1111/j.1745-493X.2009.03174.x 
Bocken, N. M. P.; Allwood, J. M.; Willey, A. R.; King, J. M. H. Development of a tool for rapidly assessing the implementation difficulty and emissions benefits of innovations.

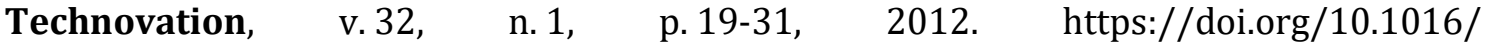
j.technovation.2011.09.005

Brunner, P. H.; Rechberger, H. Handbook of material flow analysis: For environmental, resource, and waste engineers. 2. ed. New York: Taylor e Francis Group CRC press, 2016.

Cao, B.; Xiao, Z.; Li, X. Joint decision on pricing and waste emission level in industrial symbiosis chain. Journal of Industrial and Management Optimization, v. 14, n. 1, p. 135-164, 2018. https://doi.org/10.3934/jimo.2017040

Chertow, M.; Park, J. Scholarship and practice in industrial symbiosis: 1989-2014. In: Clift, R.; Druckman, A. (Eds.). Taking Stock of Industrial Ecology. Cham: Springer, 2016. p. 87-116. https://doi.org/10.1007/978-3-319-20571-7_5

Chertow, M. R. Industrial symbiosis: Literature and taxonomy. Annual Review of Energy and the Environment, $\quad$ v. 25, n. 1, p. 313-337, 2000. https://doi.org/10.1146/ annurev.energy.25.1.313

Chertow, M. R. "Uncovering” industrial symbiosis. Journal of Industrial Ecology, v. 11, n. 1, p. 11-30, 2007. https://doi.org/10.1162/jiec.2007.1110

Despeisse, M.; Ball, P. D.; Evans, S.; Levers, A. Industrial ecology at factory level: A conceptual model. Journal of Cleaner Production, v. 31, p. 30-39, 2012. https://doi.org/10.1016/j.jclepro.2012.02.027

Doménech, T.; Davies, M. The role of embeddedness in industrial symbiosis networks: phases in the evolution of industrial symbiosis networks. Business Strategy and the Environment, v. 20, n. 5, p. 281-296, 2011. https://doi.org/10.1002/bse.695

$\mathrm{Du}, \mathrm{S}$;; Hu, L.; Wang, L. Low-carbon supply policies and supply chain performance with carbon concerned demand. Annals of Operations Research, v. 255, n. 1/2, p. 569-590, 2017. https://doi.org/10.1007/s10479-015-1988-0

Eckelman, M. J.; Chertow, M. R. Quantifying life cycle environmental benefits from the reuse of industrial materials in Pennsylvania. Environmental Science \& Technology, v. 43, n. 7, p. 2550-2556, 2009. https://doi.org/10.1021/es802345a

Fernandes, J. L.; Qualharini, E. L.; Fernandes, A. S. C.; Nóbrega, M. J. R. Um estudo da produção mais limpa na gestão ambiental. Revista Augustus, v. 20, n. 39, p. 52-64, 2015.

Fernandes, J. V. G.; Gonçalves, E.; Andrade, J. C. S.; Kiperstok, A. Introduzindo práticas de produção mais limpa em sistemas de gestão ambiental certificáveis: uma proposta prática. Engenharia Sanitária e Ambiental, v. 6, n. 3, p. 157-164, 2001.

FIEMG - Federação das Indústrias do Estado de Minas Gerais. Programa Mineiro de Simbiose Industrial - PMSI. Belo Horizonte: FIEMG, 2014. Disponível em: <http://www.fiemg.org.br/Default.aspx?tabid=10992>. Acesso: 04 jun. 2018.

Gil, A. C. Como elaborar projetos de pesquisa. São Paulo: Atlas, 1996.

Goodman, L. A. Snowball sampling. The Annals of Mathematical Statistics, v. 32, n. 1, p. 148-170, 1961. https://doi.org/10.1214/aoms/1177705148

Grant, R. F.; Barr, A. G.; Black, T. A.; Margolis, H. A.; McCaughey, J. H.; Trofymow, J. A. Net ecosystem productivity of temperate and boreal forests after clearcutting: A FluxnetCanada measurement and modelling synthesis. Tellus B: Chemical and Physical Meteorology, $\quad$ v. 62, $\quad$ n. 5, p. 475-496, 2010. https://doi.org/10.1111/j.16000889.2010.00500.x 
IBGE - Instituto Brasileiro de Geografia e Estatística. Pesquisa Nacional de Saneamento Básico. Rio de Janeiro: IBGE, 2017. Disponível em: <http://ibgealllingerie.com.br/ pesquisa-nacional-de-saneamento-básico/>. Acesso em: 10 abr. 2018.

Jacobsen, N. B. Industrial symbiosis in Kalundborg, Denmark: A quantitative assessment of economic and environmental aspects. Journal of Industrial Ecology, v. 10, n. 1/2, p. 239-255, 2006. https://doi.org/10.1162/108819806775545411

Lowe, E. A.; Evans, L. K. Industrial ecology and industrial ecosystems. Journal of Cleaner Production, v. 3, n. 1/2, p. 47-53, 1995. https://doi.org/10.1016/0959-6526(95)00045-G

Maroun, M.; Richter-Levin, G. Exposure to acute stress blocks the induction of long-term potentiation of the amygdala-prefrontal cortex pathway in vivo. Journal of Neuroscience, v. 23, n. 11, p. 4406-4409, 2003. https://doi.org/10.1523/jneurosci.23-11-04406.2003

Mattar, F. N. Pesquisa de marketing. São Paulo: Atlas, 1999. v. 6.

Miguel, P. A. C. Estudo de caso na engenharia de produção: estruturação e recomendações

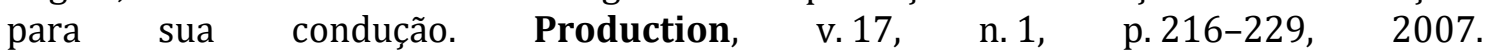
https://doi.org/10.1590/S0103-65132007000100015

Mirata, M.; Pearce, R. Industrial symbiosis in the UK. In: Green, I. K.; Randles, S. (Eds.). Industrial Ecology and spaces of innovation. Bodmin: MPG Books, 2006. p. 77-106.

Moher, D.; Liberati, A.; Tetzlaff, J.; Altman, D. G.; Altman, D.; Antes, G.; Clark, J. Preferred reporting items for systematic reviews and meta-analyses: The PRISMA statement (Chinese edition). Journal of Chinese Integrative Medicine, v. 7, n. 9, p. 889-896, 2009. https://doi.org/10.3736/jcim20090918

OECD - Organization for Economic Cooperation and Development. 2017. Disponível em: <http://www.oecd.org>. Acesso: 10 abr. 2018.

Ohnishi, S.; Fujita, T.; Chen, X.; Fujii, M. Econometric analysis of the performance of recycling projects in Japanese Eco-Towns. Journal of Cleaner Production, v. 33, p. 217-225, 2012. https://doi.org/10.1016/j.jclepro.2012.03.027

Paiva, F. F. G.; Maria, V. P. K. Gestão ambiental de resíduos industriais: análise de gestão e reaproveitamento de resíduos da indústria sucroalcooleira. Revista Brasileira de Gestão Ambiental e Sustentabilidade, v. 5, n. 9, p. 157-166, 2018. https://doi.org/10.21438/ rbgas. 050910

Queiroz, N. T.; Vieira, E. T. V. Gestão de resíduos sólidos na zona urbana do Município de Varzelândia, Minas Gerais, Brasil: um olhar pela via da gestão municipal e impressões da população. Revista Brasileira de Gestão Ambiental e Sustentabilidade, v. 5, n. 9, p. 141-156, 2018. https://doi.org/10.21438/rbgas.050909

Rodrigues, T. V.; Jesus, R. G.; Oliveira, N. P. A importância do gerenciamento de projetos para pequenas e médias empresas. Gestão e Desenvolvimento em Revista, v. 5, n. 1, p. 4-12, 2019.

Santos, I. E. Manual de métodos e técnicas de pesquisa científica. Niteroi: Impetus, 2005.

Santos, V. E. N.; Magrini, A. Biorefining and industrial symbiosis: A proposal for regional development in Brazil. Journal of Cleaner Production, v. 177, p. 19-33, 2018. https://doi.org/10.1016/j.jclepro.2017.12.107

Schott, G. L. M.; Vasconcelos, F.C.W. Manual para implementação da gestão socioambiental dos resíduos sólidos têxteis. Blucher Design Proceedings, v. 2, n. 9, p. 3239-3249, 2016. 
Sheldon, R. A. Metrics of green chemistry and sustainability: Past, present, and future. ACS Sustainable Chemistry \& Engineering, v. 6, n. 1, p. 32-48, 2018. https://doi.org/ 10.1021/acssuschemeng.7b03505

Vergara, S. C.; Peci, A. Escolhas metodológicas em estudos organizacionais. Organizações $\begin{array}{llllll}\& & \text { Sociedade, } & \text { v. } 10, & \text { n. } 27, & \text { p. 13-26, 2003. https://doi.org/10.1590/S1984- }\end{array}$ 92302003000300001

Yin, R. K. Estudo de caso: planejamento e métodos. Porto Alegre: Bookman, 2015. 